\title{
Random-Walk Term Weighting for Improved Text Classification
}

\author{
Samer Hassan and Carmen Banea \\ Department of Computer Science \\ University of North Texas \\ Denton, TX 76203 \\ samer@unt.edu, carmen@unt.edu
}

\begin{abstract}
This paper describes a new approach for estimating term weights in a text classification task. The approach uses term cooccurrence as a measure of dependency between word features. A random walk model is applied on a graph encoding words and co-occurrence dependencies, resulting in scores that represent a quantification of how a particular word feature contributes to a given context. We argue that by modeling feature weights using these scores, as opposed to the traditional frequency-based scores, we can achieve better results in a text classification task. Experiments performed on four standard classification datasets show that the new random-walk based approach outperforms the traditional term frequency approach to feature weighting.
\end{abstract}

\section{Introduction}

Term frequency has long been adapted as a measure of term significance in a specific context (Robertson and Jones, 1997). The logic behind it is that the more a certain term is encountered in a certain context, the more it carries or contributes to the meaning of the context. Due to this belief, term frequency has been a major factor in estimating the probabilistic distribution of features using maximum likelihood estimates and hence has been incorporated in a broad spectrum of tasks ranging from feature selec- tion techniques (Yang and Pedersen, 1997; Schutze et al., 1995) to language models (Bahl et al., 1983).

In this paper we introduce a new measure of term weighting, which integrates the locality of a term and its relation to the surrounding context. We model this local contribution using a co-occurrence relation in which terms that co-occur in a certain context are likely to share between them some of their importance (or significance). Note that in this model the relation between a given term and its context is not linear, since the context itself consists of a collection of other terms, which in turn have a dependency relation with their own context, which might include the original given term. In order to model this recursive relation we use a graph-based ranking algorithm, namely the PageRank randomwalk algorithms (Brin and Page, 1998), and its TextRank adaption to text processing applications (Mihalcea and Tarau, 2004). TextRank takes as input a set of textual entities and relations between them, and uses a graph-based ranking algorithm (also known as random walk algorithm) to produce a set of scores that represent the accumulated weight or rank for each textual entity in their context. The TextRank model was so far evaluated on three natural language processing tasks: document summarization, word sense disambiguation, and keyword extraction, and despite being fully unsupervised, it has been shown to be competitive with other sometime supervised state-of-the-art algorithms.

In this paper, we show how TextRank can be used to model the probabilistic distribution of word features in a document, by making further use of the scores produced by the random-walk model. 
Through experiments performed on a text classification task, we show that these random walk scores outperform the traditional term frequencies typically used to model the feature weights for this task.

\section{Graph-based Ranking Algorithms}

The basic idea implemented by an iterative graphbased ranking algorithm is that of "voting" or "recommendation". When one vertex links to another one, it is basically casting a vote for that other vertex. The higher the number of votes that are cast for a vertex, the higher the importance of the vertex. Moreover, the importance of the vertex casting a vote determines how important the vote itself is, and this information is also taken into account by the ranking algorithm. Hence, the score associated with a vertex is determined based on the votes that are cast for it, and the scores of the vertices casting these votes.

While there are several graph-based ranking algorithms previously proposed in the literature (Herings et al., 2001), we focus on only one such algorithm, namely PageRank (Brin and Page, 1998), as it was previously found successful in a number of applications, including Web link analysis (Brin and Page, 1998), social networks (Dom et al., 2003), citation analysis, and more recently in several text processing applications (Mihalcea and Tarau, 2004), (Erkan and Radev, 2004).

Given a graph $G=(V, E)$, let $\operatorname{In}\left(V_{a}\right)$ be the set of vertices that point to vertex $V_{a}$ (predecessors), and let $\operatorname{Out}\left(V_{a}\right)$ be the set of vertices that vertex $V_{a}$ points to (successors). The PageRank score associated with the vertex $V_{a}$ is then defined using a recursive function that integrates the scores of its predecessors:

$$
S\left(V_{a}\right)=(1-d)+d * \sum_{V_{b} \in \operatorname{In}\left(V_{a}\right)} \frac{S\left(V_{b}\right)}{\left|\operatorname{Out}\left(V_{b}\right)\right|}
$$

where $d$ is a parameter that is set between 0 and $1^{1}$.

The score of each vertex is recalculated upon each iteration based on the new weights that the neighboring vertices have accumulated. The algorithm terminates when the convergence point is reached for all the vertices, meaning that the error rate for each vertex falls below a pre-defined threshold. Formally,

\footnotetext{
${ }^{1}$ The typical value for $d$ is 0.85 (Brin and Page, 1998), and this is the value we are also using in our implementation.
}

for a vertex $V_{i}$ let $S^{k}\left(V_{i}\right)$ be the rank or the score at iteration $k$ and $S^{k+1}\left(V_{i}\right)$ be the score at iteration $k+1$. The error rate $E R$ is defined as:

$$
E R=S^{k+1}\left(V_{i}\right)-S^{k}\left(V_{i}\right)
$$

This vertex scoring scheme is based on a random walk model, where a walker takes random steps on the graph $G$, with the walk being modeled as a Markov process - that is, the decision on what edge to follow is solely based on the vertex where the walker is currently located. Under certain conditions, this model converges to a stationary distribution of probabilities, associated with vertices in the graph. Based on the Ergodic theorem for Markov chains (Grimmett and Stirzaker, 1989), the algorithm is guaranteed to converge if the graph is both aperiodic and irreducible. The first condition is achieved for any graph that is a non-bipartite graph, while the second condition holds for any strongly connected graph - property achieved by PageRank through the random jumps introduced by the $(1-d)$ factor. In matrix notation, the PageRank vector of stationary probabilities is the principal eigenvector for the matrix $A_{\text {row }}$, which is obtained from the adjacency matrix $A$ representing the graph, with all rows normalized to sum to 1 : $\left(P=A_{\text {row }}^{T} P\right)$.

Intuitively, the stationary probability associated with a vertex in the graph represents the probability of finding the walker at that vertex during the random walk, and thus it represents the importance of the vertex within the graph. In the context of sequence data labeling, the random walk is performed on the label graph associated with a sequence of words, and thus the resulting stationary distribution of probabilities can be used to decide on the most probable set of labels for the given sequence.

\subsection{TextRank}

Given a natural language processing task, the TextRank model includes four general steps for the application of a graph-based ranking algorithm to graph structures derived from natural language texts:

1. Identify text units that best define the proposed task and add them as vertices in the graph.

2. Identify relations that connect such test units, and use these relations to draw edges between 
vertices in the graph. Edges can be directed or undirected, weighted or un-weighted.

3. Iterate the graph ranking algorithm to convergence.

4. Sort vertices based on their final score. Use the values attached to each vertex for ranking.

The strength of this model lies in the global representation of the context and its ability to model how the co-occurrence between features might propagate across the context and affect other distant features.

While TextRank has already been applied to several language processing tasks, we focus here on the keyword extraction task, since it best relates to our approach. The goal of a keyword extraction tool is to find a set of words or phrases that best describe a given document. The co-occurrence relation within a specific window is used to portray the correlation between words, which are represented as vertices in the graph. Two vertices are connected if their corresponding lexical units co-occur within a window of at most $N$ words, where $N$ can be set to any value greater than two. The TextRank application to keyword extraction has also used different syntactic filters for vertex selection, including all open class words, nouns and verbs, nouns and adjectives, and others. The algorithm was found to provide the best results using nouns and adjectives with a window size of two.

Our approach follows the same main steps as used in the TextRank keyword extraction application. We are however incorporating a larger number of lexical units, and we use different window sizes, as we will show in the following section.

\section{TextRank for Term Weighting}

The goal of the work reported in this paper is to study the ranking scores obtained using TextRank, and evaluate their potential usefulness as a new measure of term weighting.

To understand how the random-walk weights (rw) might be a good replacement for the traditional term frequency weights $(t f)$, consider the example in Figure 1. The example represents a sample document from the Reuters collection. A graph is constructed as follows. If a term has not been previously seen, then a node is added to the graph to represent this term. A term can only be represented by one node in the graph. An undirected edge is drawn between two nodes if they co-occur within a certain window size. This example assumes a window size of two, corresponding to two consecutive terms in the text (e.g. London is linked to based).

London-based sugar operator Kaines Ltd confirmed it sold two cargoes of white sugar to India out of an estimated overall sales total of four or five cargoes in which other brokers participated. The sugar, for April/May and April/June shipment, was sold at between 214 and 218 dlrs a tonne cif, it said.

Figure 1: Sample Reuters document

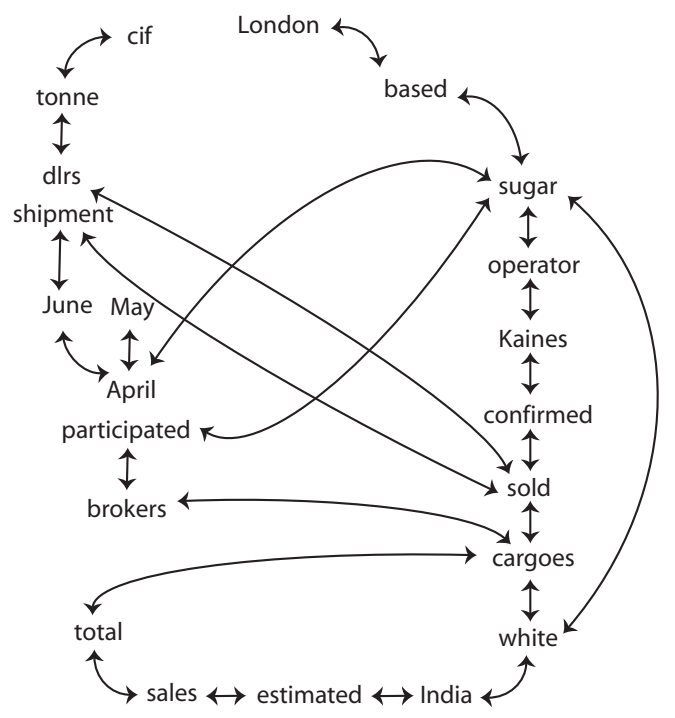

Figure 2: Sample graph

Table 1 shows the $t f$ and $r w$ weights, also plotted in Figure 3. By analyzing the $r w$ weights, we can observe a non-linear correlation with the $t f$ weights, with an emphasis given to terms surrounding important key term like e.g. "sugar" or "cargoes." This spatial locality has resulted in higher ranks for terms like "operator" compared to other terms like "london" 2 .

\footnotetext{
${ }^{2}$ All the missing words (e.g. "Ltd," "it") that are not shown in the graph are common-words that were eliminated in the preprocessing phase.
} 


\begin{tabular}{l|l|l} 
Term & $r w$ & $t f$ \\
\hline sugar & 2.248 & 3 \\
sold & 1.594 & 2 \\
april & 1.407 & 2 \\
cargoes & 1.542 & 2 \\
cif & 0.600 & 1 \\
sales & 0.891 & 1 \\
london & 0.546 & 1 \\
tonne & 1.059 & 1 \\
shipment & 0.829 & 1 \\
based & 0.933 & 1 \\
estimated & 0.888 & 1 \\
dlrs & 0.938 & 1 \\
kaines & 0.871 & 1 \\
confirmed & 0.859 & 1 \\
total & 0.856 & 1 \\
white & 0.796 & 1 \\
india & 0.846 & 1 \\
operator & 0.839 & 1 \\
brokers & 0.826 & 1 \\
june & 0.801 & 1 \\
participated & 0.819 & 1
\end{tabular}

Table 1: $t f \& r w$ scores

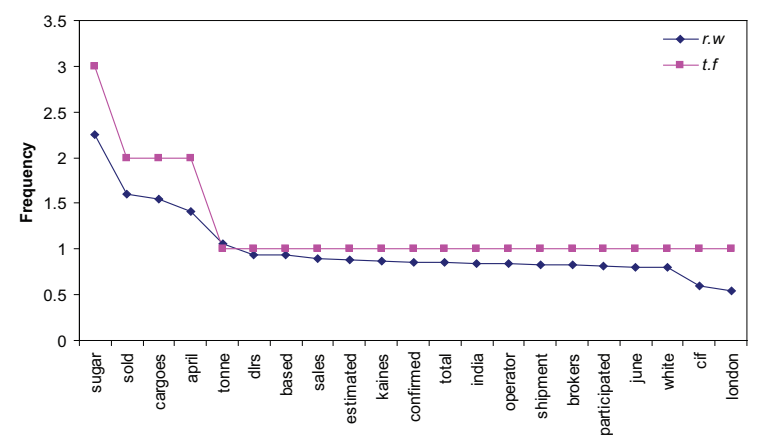

Figure 3: $t f \& r w$ plots

\section{Experimental Setup}

To evaluate our random-walk based approach to feature weighting, we integrate it in a text classification algorithm, and evaluate its performance on several standard text classification data sets.

\subsection{Random-Walk Term Weighting}

Starting with a given document, we determine a ranking over the words in the document by using the approach described in Section 3.

First, we tokenize the document for punctuation, special symbols, word abbreviations. We also remove the common words, using a list of approximately 500 frequently used words as used in the
Smart retrieval system ${ }^{3}$.

Next, the resulting text is processed to extract both $t f$ and $r w$ weights for each term in the document. Note that we do not apply any syntactic filters, as it was previously done in applications of TextRank. Instead, we consider each word as a potential feature. To determine $t f$ we simply count the frequencies of each word in the document. To determine $r w$, all the terms are added as vertices in a graph representing the document. A co-occurrence scanner is then applied to the text to relate the terms that co-occur within a given window size . For a given term, all the terms that fall in the vicinity of this term are considered dependent terms. This is represented by a set of edges that connect this term to all the other terms in the window. Experiments are performed for window sizes of 2, 4, 6, and 8 . Once the graph is constructed and the edges are in place, the TextRank algorithm is applied ${ }^{4}$. The result of the ranking process is a list of all input terms and their corresponding $r w$ scores.

We then calculate $t f . i d f$ and $r w . i d f$ as follows:

$$
t f . i d f=t f * \log \frac{N_{D}}{n}
$$

where $N_{D}$ represent the total number of documents in the collection and $n$ is the number of documents in which the target term appeared at least once.

Similarly,

$$
r w . i d f=r w * \log \frac{N_{D}}{n}
$$

These term weights ( $t f . i d f$ or $r w . i d f)$ are then used to create a feature vector for each document. The vectors are fed to a traditional text classification system, using one of the learning algorithms described below. The results obtained using $t f$.idf will act as a baseline in our evaluation.

\subsection{Text Classification}

Text classification is a problem typically formulated as a machine learning task, where a classifier learns how to distinguish between categories in a given set

\footnotetext{
${ }^{3} \mathrm{ftp}: / / \mathrm{ftp} . c s . c o r n e l l . e d u / p u b / s m a r t$.

${ }^{4}$ We use an implementation where the maximum number of iterations is limited to 100 , the damping factor is set to 0.85 , and convergence threshold to 0.0001 . Each graph node is assigned with an initial weight of 0.25 .
} 
using features automatically extracted from a collection of training documents. There is a large body of algorithms previously tested on text classification problems, due also to the fact that this task is one of the testbeds of choice for machine learning algorithms. In the experiments reported here, we compare results obtained with four frequently used text classifiers - Rocchio, Naïve Bayes, Nearest Neighbor, and Support Vector Machines, selected based on their diversity of learning methodologies.

Naïve Bayes. The basic idea in a Naïve Bayes text classifier is to estimate the probability of a category given a document using joint probabilities of words and documents. Naïve Bayes assumes word independence, which means that the conditional probability of a word given a category is assumed to be independent of the conditional probability of other words given the same category. Despite this simplification, Naïve Bayes classifiers were shown to perform surprisingly well on text classification (Joachims, 1997), (Schneider, 2004). While there are several versions of Naïve Bayes classifiers (variations of multinomial and multivariate Bernoulli), we use the multinomial model (McCallum and Nigam, 1998), which was shown to be more effective.

Rocchio. This is an adaptation of the relevance feedback method developed in information retrieval (Rocchio, 1971). It uses standard $t f$.idf weighted vectors to represent documents, and builds a prototype vector for each category by summing up the vectors of the training documents in each category. Test documents are then assigned to the category that has the closest prototype vector, based on a cosine similarity. Text classification experiments with different versions of the Rocchio algorithm showed competitive results on standard benchmarks (Joachims, 1997), (Moschitti, 2003).

KNN. K-Nearest Neighbor is one of the earliest text categorization approaches (Makoto and Takenobu, 1995; Masand et al., 1992). The algorithm classifies a test document based on the best class label identified for the nearest K-neighbors in the training documents. The best class label is chosen by weighting the class of each similar training document with its similarity to the target test document.

SVM. Support Vector Machines (Vapnik, 1995) is a state-of-the-art machine learning approach based on decision plans. The algorithm defines the best hyper-plan which separates set of points associated with different class labels with a maximum-margin. The unlabeled examples are then classified by deciding in which side of the hyper-surface they reside. The hyper-plan can be a simple linear plan as first proposed by Vapnik, or a non-linear plan such as e.g. polynomial, radial, or sigmoid. In our evaluation we used the linear kernel since it was proved to be as powerful as the other kernels when tested on text classification data sets (Yang and Liu, 1999).

\subsection{Data Sets}

In our experiments we use Reuters-21578, WebKB, 20Newsgroups, and LingSpam datasets. These datasets are commonly used for text classification evaluations (Joachims, 1996; Craven et al., 1998; Androutsopoulos et al., 2000; Mihalcea and Hassan, 2005).

Reuter-21578. This is a publicly available subset of the Reuters news, containing about 120 categories. We use the standard ModApte data split (Apte et al., 1994). The unlabeled documents were discarded and only the documents with one or more class labels were used in the classification experiments.

WebKB. This is a data set collected from computer science departments of various universities by the CMU text learning group. The dataset contains seven class labels which are Project, Student, Department, Faculty, Staff, Course, and Other. The Other label was removed from the dataset for evaluation purposes. Most of the evaluations in the literature have been performed on only four of the categories (Project, Student, Faculty, and Course) since they represent the largest categories. However, since we wanted to see how our system behaves when only a few training examples were available as e.g. in the Staff and the Department classes, we performed our evaluations on two versions of $W e b K B$ : one with the four categories version $\left(W e b K B_{4}\right)$ and one with the six categories $\left(W e b K B_{6}\right)$.

20-Newsgroups. This is a collection of 20,000 messages from 20 different newsgroups, corresponding to different topics or subjects. Each newsgroup has about 1000 message split into 400 test and 600 train documents.

LingSpam. This is a spam corpus, consisting of email messages organized in 10 collections to al- 
low for 10-fold cross validation. Each collection has roughly 300 spam and legitimate messages. There are four versions of the corpus standing for bare, stop-word filtered, lemmatized, and stop-word and lemmatized. We use the bare collection with a standard 10-fold cross validation.

\subsection{Performance Measures}

To evaluate the classification system we used the traditional accuracy measure defined as the number of correct predictions divided with the number of evaluated examples.

We also use the correlation coefficient $(\rho)$ as a diversity measure to evaluate the dissimilarity between the weighting models. Pairwise diversity measures have been traditionally used to measure the statistical independence among ensemble of classifiers (Kuncheva and Whitaker, 2003). Here, we use them to measure the correlation between our random-walk approach and the traditional term frequency approach. The typical setting in which the pairwise diversity measures are used is a set of different classifiers which are used to classify the same set of feature vectors or documents over a given dataset. In our evaluation we use the same classifier to evaluate two different sets of feature vectors that are produced by different weighting features: the $r w$ random walk weighting, and the $t f$ term frequency weighting. Since the two feature vector collections are evaluated by one classifier at a time, the resulted diversity scores will reflect the diversity of the two systems.

Let $D_{i}$ and $D_{j}$ be two feature weighting models with the following contingency table.

\begin{tabular}{|l|l|l|}
\hline & $D_{j \text { correct }}=Y$ & $D_{j \text { correct }}=N$ \\
\hline$D_{i \text { correct }}=$ & $a$ & $\mathrm{~b}$ \\
\hline$D_{i \text { correct }}=N$ & $c$ & $\mathrm{~d}$ \\
\hline
\end{tabular}

Table 2: $D_{i} \& D_{j}$ Contingency table

The correlation coefficient $(\rho)$ is defined as:

$$
\rho_{i j}=\frac{a d-b c}{\sqrt{(a+b)(c+d)(a+c)(b+d)}}
$$

\footnotetext{
${ }^{5}$ The symbol $†$ indicates a statistically significant result using
}

Table 3: Naive Bayes Results ${ }^{5}$

\begin{tabular}{|l|l|l|l|l|l|}
\hline N.B. & $t f$ & $r w_{2}$ & $r w_{4}$ & $r w_{6}$ & $r w_{8}$ \\
\hline WebKB $B_{4}$ & 81.9 & 81.9 & $\mathbf{8 2 . 8}$ & 82.7 & 81.2 \\
\hline WebKB $B_{6}$ & 71.7 & 73.0 & $74.2 \dagger$ & $\mathbf{7 4 . 4} \dagger$ & 73.5 \\
\hline Reuter & $\mathbf{8 3 . 2}$ & 82.5 & 82.9 & 83.0 & 82.8 \\
\hline $20 N G$ & 81.7 & 82.0 & $\mathbf{8 2 . 3} \ddagger$ & $82.3 \ddagger$ & $82.1 \dagger$ \\
\hline LSpam & 99.3 & $\mathbf{9 9 . 4}$ & 99.3 & 99.3 & 99.3 \\
\hline
\end{tabular}

Table 4: Rocchio Results

\begin{tabular}{|l|l|l|l|l|l|}
\hline ROC & $t f$ & $r w_{2}$ & $r w_{4}$ & $r w_{6}$ & $r w_{8}$ \\
\hline WebKB $B_{4}$ & 71.9 & $77.5 \ddagger$ & $\mathbf{7 8 . 6} \ddagger$ & $80.8 \ddagger$ & $80.9 \ddagger$ \\
\hline WebKB $B_{6}$ & 58.3 & $69.6 \ddagger$ & $72.0 \ddagger$ & $\mathbf{7 6 . 5}$ & $76.2 \ddagger$ \\
\hline Reuter & 78.2 & $80.8 \ddagger$ & $81.1 \ddagger$ & $81.0 \ddagger$ & $\mathbf{8 1 . 4} \ddagger$ \\
\hline $20 N G$ & 76.2 & $77.3 \ddagger$ & $77.1 \ddagger$ & $77.2 \ddagger$ & $\mathbf{7 7 . 4} \ddagger$ \\
\hline LSpam & 97.5 & $\mathbf{9 7 . 8}$ & 97.8 & 97.7 & 97.8 \\
\hline
\end{tabular}

\section{Evaluation and Discussion}

Tables 3, 4, 5, 6 show the classification results for $W e b K B_{4}$, WebKB $B_{6}$, LingSpam, Reuter, and $20 N$ ewsgroups respectively. The $r w_{2}, r w_{4}, r w_{6}$, and $r w_{8}$ represent the accuracies achieved using random-walk weighting under window sizes of 2 , 4,6 , and 8 respectively. The $t f$ column represents the results obtained with a term frequency weighting scheme.

By examining the results we can see that the $r w . i d f$ model outperforms the $t f . i d f$ model on all the classifiers and datasets with only one exception in the case of a Naïve Bayes classifier under Reuter. The error reductions range from $3.5 \%$ as in $\left\{20\right.$ Newsgroups, NaiveBayes, $\left.r w_{4}\right\}$ to $44 \%$ as in the case of $\left\{W e b K B_{6}\right.$, Rocchio, $\left.r w_{6}\right\}$. The system gives, in its worst performance, a comparable result to the $t f . i d f$ baseline. The system shows a consistent performance with different window sizes, with no clear cut window size that would give the best result. By further analyzing the results using statistical paired t-tests we can see that windows of size 4 and 6 supply the most significant results across all the classifiers as well as the datasets.

Comparing $W e b K B_{4}$ and $W e b K B_{6}$ fine-grained results, we found that both systems failed to predict the class Staff; however the significant improve-

a paired t-test, with $p<0.05$. The result is marked by $\ddagger$ when $p<0.001$. 
Table 5: KNN Results

\begin{tabular}{|l|l|l|l|l|l|}
\hline$K N N$ & $t f$ & $r w_{2}$ & $r w_{4}$ & $r w_{6}$ & $r w_{8}$ \\
\hline WebKB $B_{4}$ & 59.2 & $\mathbf{6 8 . 6}$ & $67.0 \ddagger$ & $64.6 \ddagger$ & $66.6 \ddagger$ \\
\hline WebK $B_{6}$ & 55.8 & $\mathbf{6 3 . 7} \ddagger$ & 55.8 & $59.9 \dagger$ & $61.0 \ddagger$ \\
\hline Reuter & 73.6 & $76.9 \ddagger$ & $78.1 \ddagger$ & $\mathbf{7 8 . 5} \ddagger$ & $78.5 \ddagger$ \\
\hline $20 N G$ & 70.3 & $76.1 \ddagger$ & $76.5 \ddagger$ & $77.2 \ddagger$ & $\mathbf{7 7 . 8} \ddagger$ \\
\hline$L S p a m$ & 97.5 & 97.8 & 97.8 & $\mathbf{9 8 . 1} \dagger$ & 97.9 \\
\hline
\end{tabular}

Table 6: SVM Results

\begin{tabular}{|l|l|l|l|l|l|}
\hline$S V M$ & $t f$ & $r w_{2}$ & $r w_{4}$ & $r w_{6}$ & $r w_{8}$ \\
\hline WebKB $B_{4}$ & 87.7 & 87.9 & 87.9 & $\mathbf{8 9} \dagger$ & 88.5 \\
\hline WebKB $B_{6}$ & 82.5 & $84.5 \ddagger$ & $\mathbf{8 5 . 2} \ddagger$ & $85.2 \ddagger$ & $84.6 \ddagger$ \\
\hline Reuter & 83.2 & $84.5 \ddagger$ & $84.4 \ddagger$ & $\mathbf{8 4 . 6}$ & $84.1 \dagger$ \\
\hline $20 N G$ & 95.2 & $95.5 \ddagger$ & $\mathbf{9 5 . 6}$ & $95.6 \ddagger$ & $95.4 \dagger$ \\
\hline LSpam & 95.6 & $96.4 \ddagger$ & $\mathbf{9 6 . 4} \ddagger$ & $96.2 \ddagger$ & $96.3 \ddagger$ \\
\hline
\end{tabular}

ment was over the class Department, in which our $r w$ model scores an accuracy of $47 \%$ compared to $4 \%$ in using $t f . i d f$. This indicates how successful $r w . i d f$ model is in cases where there are few training examples. This could be due to the ability of the model to extract more realistic and smoother distribution of terms as seen in the $r w$ curve plotted in Figure 3, hence reducing the feature bias imposed by the limited number of training examples.

Table 7: Naive Bayes Correlation $\rho$

\begin{tabular}{|l|c|c|c|c|}
\hline N.B. & $r w_{2}$ & $r w_{4}$ & $r w_{6}$ & $r w_{8}$ \\
\hline WebKB & 0.68 & 0.70 & 0.70 & $\mathbf{0 . 6 6}$ \\
\hline WebKB & 0.71 & 0.71 & 0.71 & $\mathbf{0 . 6 5}$ \\
\hline Reuter & 0.86 & 0.87 & 0.87 & $\mathbf{0 . 8 5}$ \\
\hline $20 N G$ & $\mathbf{0 . 8 2}$ & 0.84 & 0.83 & 0.82 \\
\hline LSpam & $\mathbf{0 . 8 9}$ & 0.89 & 0.92 & 0.92 \\
\hline
\end{tabular}

By also examining the diversity of the classification systems based on $r w$ and $t f$ weighting, as shown in Table 7, 8, 9, 10, we can see an interesting property of the system. The two models are generally more diverse and less correlated when using windows of size 6 and 8 than using windows of size 2 and 4 . This could be due to the increasing drift from the feature independence assumption that is implied by $t f . i d f$. However increasing the dependency is not always desirable as seen in the reported accuracies. We expect that at a certain window size the system performance will degrade to $t f . i d f$. This
Table 8: Rocchio Correlation $\rho$

\begin{tabular}{|l|c|c|c|c|}
\hline ROC & $r w_{2}$ & $r w_{4}$ & $r w_{6}$ & $r w_{8}$ \\
\hline WebK $_{4}$ & $\mathbf{0 . 4 9}$ & 0.51 & 0.53 & 0.54 \\
\hline WebK $B_{6}$ & $\mathbf{0 . 4 0}$ & 0.40 & 0.41 & 0.42 \\
\hline Reuter & 0.75 & 0.77 & 0.75 & $\mathbf{0 . 7 1}$ \\
\hline $20 N G$ & 0.77 & 0.77 & 0.77 & $\mathbf{0 . 7 7}$ \\
\hline LSpam & 0.82 & 0.85 & 0.81 & $\mathbf{0 . 7 8}$ \\
\hline
\end{tabular}

Table 9: KNN Correlation $\rho$

\begin{tabular}{|l|l|l|l|l|}
\hline$K N N$ & $r w_{2}$ & $r w_{4}$ & $r w_{6}$ & $r w_{8}$ \\
\hline WebKB $B_{4}$ & 0.35 & $\mathbf{0 . 3 2}$ & 0.36 & 0.37 \\
\hline WebKB & $\mathbf{0 . 3 5}$ & 0.35 & 0.37 & 0.37 \\
\hline Reuter & 0.74 & 0.70 & 0.68 & $\mathbf{0 . 6 7}$ \\
\hline $20 N G$ & 0.62 & 0.64 & 0.63 & $\mathbf{0 . 5 9}$ \\
\hline LSpam & 0.66 & 0.69 & 0.63 & $\mathbf{0 . 5 7}$ \\
\hline
\end{tabular}

threshold window size will be equal to the document size. In such a case each term will depend on all the remaining terms resulting in an almost completely connected graph. Consequently, each feature contribution to the surrounding will be equal resulting in similar $r w$ scores to all the features.

\section{Conclusions and Future Work}

Based on results obtained in text classification experiments, the TextRank random-walk model to term weighting was found to achieve error rate reductions of $3.5-44 \%$ as compared to the traditional frequency-based approach. The evaluation results have shown that the system performance varies depending on window size, dataset, as well as classifier, with the greatest boost in performance recorded for KNN ,Rocchio, and SVM. We believe that these results support our claim that random-walk models can accurately estimate term weights, and can be used as a technique to model the probabilistic distribution of features in a document.

The evaluations reported in this paper has shown that the TextRank model can accurately provide unigram probabilities for a sequence of words. In future work we will try to extend the TextRank model and use it to define a formal language model in which we can estimate the probability of entire sequences of words (n-grams). 
Table 10: SVM Correlation $\rho$

\begin{tabular}{|l|c|c|c|c|}
\hline$S V M$ & $r w_{2}$ & $r w_{4}$ & $r w_{6}$ & $r w_{8}$ \\
\hline WebKB & $\mathbf{0 . 7 3}$ & 0.77 & 0.78 & 0.82 \\
\hline WebKB & $\mathbf{0 . 7 3}$ & 0.76 & 0.78 & 0.80 \\
\hline Reuter & $\mathbf{0 . 8 0}$ & 0.83 & 0.82 & 0.82 \\
\hline $20 N G$ & 0.80 & $\mathbf{0 . 7 8}$ & 0.82 & 0.83 \\
\hline LSpam & $\mathbf{0 . 8 6}$ & 0.88 & 0.88 & 0.89 \\
\hline
\end{tabular}

\section{References}

I. Androutsopoulos, J. Koutsias, K. V. Chandrinos, G. Paliouras, and C. D. Spyropoulos. 2000. An evaluation of naive bayesian anti-spam filtering. In Proceedings of the workshop on Machine Learning in the New Information Age.

C. Apte, F. Damerau, and S. M. Weiss. 1994. Towards language independent automated learning of text categorisation models. In Proceedings of the 17th ACM SIGIR Conference on Research and Development in Information Retrieval.

L. Bahl, F. Jelinek, and R. Mercer. 1983. A maximum likelihood approach to continuous speech recognition. IEEE Transactions on Pattern Analysis and Machine Intelligence, 5(2).

S. Brin and L. Page. 1998. The anatomy of a large-scale hypertextual Web search engine. Computer Networks and ISDN Systems, 30(1-7).

M. Craven, D. DiPasquo, D. Freitag, A. McCallum, T. Mitchell, K. Nigam, and S. Slattery. 1998. Learning to extract symbolic knowledge from the World Wide Web. In Proceedings of the 15th Conference of the American Association for Artificial Intelligence.

B. Dom, I. Eiron, A. Cozzi, and Y. Shang. 2003. Graphbased ranking algorithms for e-mail expertise analysis. In Proceedings of the 8th ACM SIGMOD workshop on Research issues in data mining and knowledge discovery, San Diego, California.

G. Erkan and D. Radev. 2004. Lexpagerank: Prestige in multi-document text summarization. In Proceedings of the Conference on Empirical Methods in Natural Language Processing, Barcelona, Spain, July.

G. Grimmett and D. Stirzaker. 1989. Probability and Random Processes. Oxford University Press.

P.J. Herings, G. van der Laan, and D. Talman. 2001. Measuring the power of nodes in digraphs. Technical report, Tinbergen Institute.

T. Joachims. 1996. A probabilistic analysis of the rocchio algorithm with tf.idf for text categorization. In Proceedings of the 14th International Conference on Machine Learning.

T. Joachims. 1997. A probabilistic analysis of the Rocchio algorithm with TFIDF for text categorization. In Proceedings of ICML-97, 14th International Conference on Machine Learning, Nashville, US.
L. Kuncheva and C. Whitaker. 2003. Measures of diversity in classifier ensembles and their relationship with the ensemble accuracy. Machine Learning, 51.

I. Makoto and T. Takenobu. 1995. Cluster-based text categorization: A comparison of category search startegies. In Proceedings of the 18th ACM International Conference on Research and Development in Information Retrieval.

B. Masand, G. Linoff, and D. Waltz. 1992. Classifying news stories using memory based reasoning. In Proceedings of the 15th International Conference on Research and Development in information Retrieval.

A. McCallum and K. Nigam. 1998. A comparison of event models for Naive Bayes text classification. In Proceedings of AAAI-98 Workshop on Learning for Text Categorization.

R. Mihalcea and S. Hassan. 2005. Using the essence of texts to improve document classification. In Proceedings of the Conference on Recent Advances in Natural Language Processing (RANLP), Borovetz, Bulgaria.

R. Mihalcea and P. Tarau. 2004. TextRank - bringing order into texts. In Proceedings of the Conference on Empirical Methods in Natural Language Processing (EMNLP 2004), Barcelona, Spain.

A. Moschitti. 2003. A study on optimal paramter tuning for Rocchio text classifier. In Proceedings of the European Conference on Information Retrieval, Pisa, Italy.

R. Robertson and K. Sparck Jones. 1997. Simple, proven approaches to text retrieval. Technical report.

J. Rocchio, 1971. Relevance feedback in information retrieval. Prentice Hall, Ing. Englewood Cliffs, New Jersey.

K. Schneider. 2004. A new feature selection score for multinomial naive bayes text classification based on kl-divergence. In The Companion Volume to the Proceedings of 42st Annual Meeting of the Association for Computational Linguistics, Barcelona, Spain, July.

H. Schutze, D. A. Hull, and J. O. Pedersen. 1995. A comparison of classifiers and document representations for the routing problem. In Proceedings of the 18th annual international ACM SIGIR conference on Research and development in information retrieval, Seattle, Washington.

V. Vapnik. 1995. The Nature of Statistical Learning Theory. Springer, New York.

Y. Yang and X. Liu. 1999. A reexamination of text categorization methods. In Proceedings of the 22nd ACM SIGIR Conference on Research and Development in Information Retrieval.

Y. Yang and J. O. Pedersen. 1997. A comparative study on feature selection in text categorization. In Proceedings of the 14th International Conference on Machine Learning, Nashville, US. 